\title{
Dominio Volitivo (*) -El dominio que faltaba-
}

Roberto J. Beltrán Neira

Profesor emérito, Profesor extraordinario investigador. Facultad de Estomatología. Universidad Peruana Cayetano Heredia.
Beltrán-Neira RJ. Dominio Volitivo -El dominio que faltaba. Rev Estomatol Herediana. 2010; 20(1):50-51.
Cuando reflexionamos sobre los alcances del dominio afectivo en el aprendizaje de la Odontología encontramos que éste no alcanza a destacar una faceta fundamental de la preparación para el ejercicio profesional del cirujano dentista (o de cualquier otro profesional), nos referimos al empeño que es necesario poner durante el aprendizaje y más tarde durante la práctica de la profesión a fin de iniciar, procesar y completar con oportunidad, eficiencia y eficacia una determinada actividad $^{1}$. El resultado de este empeño será un trabajo de calidad. A este esfuerzo que significa resistencia a la frustración, perseverancia frente a las dificultades, capacidad permanente de vigilancia crítica durante el procedimiento y repetición, cuando es necesario y posible, lo ubicamos como perteneciente al ámbito del DOMINIO VOLITIVO (de la voluntad y empeño de hacer bien al máximo de la habilidad de quien está aprendiendo o ejecutando la acción).

Partimos del supuesto de que el alumno posee el conocimiento de lo que debe ejecutar, tiene la habilidad psicomotora básica general y está favorablemente dispuesto para emprender un nuevo aprendizaje. Nos preguntamos qué se necesita para iniciar y continuar la acción hasta culminarla felizmente, creemos que se necesitan actos de voluntad, de decisión, sin los cuales ningún aprendizaje es posible. (La motivación mueve a la acción pero hace falta la decisión de responder y de esta manera iniciar la acción y continuarla hasta su conclusión).

Un ejercicio de análisis de las etapas $^{2}$ en el dominio volitivo durante la actividad psicomotora podría darnos lo siguiente:

- Rompimiento de la inercia (primer movimiento de aproximación al objeto que ha de ser motivo de la acción)

- Inicio de la operación (aplicación del primer movimiento sobre el objeto)

- Vigilancia del proceso, que a su vez comprende: voluntad de alerta bajo autocrítica severa.
- Evaluación del avance para decidir el momento terminal

- Terminación del proceso

- Evaluación del resultado

- Repetición de la acción, si siendo insuficiente es pasible de ser repetida ${ }^{3}$.

Ahora bien, si lo volitivo constituye un dominio más del aprendizaje, quiere decir que puede ser ejercitado, es decir que el alumno, poseedor de una voluntad genérica de dominar una determinada profesión, con la colaboración del instructor puede aprender a ejecutar los actos de voluntad específicos para cumplir a cabalidad las distintas etapas de la actividad que está aprendiendo. El aprendizaje de este dominio debe conducir a la instalación de un hábito de conducta permanente aplicable a la serie de actos específicos; en ello el instructor puede jugar un papel fundamental como proveedor de estímulos positivos a fin de que el alumno alcance un alto nivel de calidad. La búsqueda de excelencia en la acción terapéutica responde a un principio

(*) De: Beltrán RJ., y Beltran-Aguilar ED. Aprendizaje Intesivo a Dedicación Exclusiva. Facultad de Estomatología, Universidad Peruana Cayetano Heredia. Lima, 2002. 160 pp. (Versión revisada).

${ }^{1}$ La tercera categoría de la taxonomía "Responding" (respondiendo o respuesta) es semejante a lo que estamos llamando dominio volitivo. De ahí que vista la necesidad de destacar la importancia del aprendizaje en las sub - categorías "aquiescencia en responder" "voluntad de responder" y "satisfacción en responder" en la educación del cirujano dentista parece necesario retirar tal categoría del dominio afectivo y ubicarla en uno nuevo que proponemos como dominio volitivo. Los mismos autores de la taxonomía al referirse a la categoría "Responding", la asocian al famoso "learning by doing" (aprender mediante la acción o aprender haciendo), que nosotros vemos en el campo del aprendizaje mediante acción voluntaria antes que en el campo afectivo. Al discutir la sub-categoría "Willingness to Respond" (voluntad para responder) utilizan la palabra "voluntad" que a nuestro parecer rebasa los límites del dominio afectivo. Vayamos un poco más allá, del deseo de responder como disposición afectiva para iniciar la acción, hasta realmente iniciarla existe una brecha que creemos se cubre con el ejercicio de la voluntad que así transforma la potencia en acto. (NA)

${ }^{2}$ Preferimos llamar etapas y no sub - categorías a esta relación de componentes de la acción que exige el ejercicio de la voluntad. Por ahora las enmarcamos en las dos sub - categorías de la Taxonomía: "Aquiescencia para Responder" y "Voluntad de Responder" correspondiente a la categoría "Respuesta" y de manera difusa a las categorías "Valoración", "Organización" y "Caracterización por un Valor o Complejo de Valores" Krthwohl DR.,Bloom BS., Masia BB. Taxonomy of Educational Objectives. The Classification of Educational Goals. David McKay Company, InC. New York 1964.

${ }^{3}$ La primera etapa corresponde a "repuesta" La tercera y cuarta y sexta etapas corresponden a "valoración" Estas tres etapas son predominantemente cognitivas. No incluimos la sub - categoría "satisfacción en responder" que consideramos claramente afectiva. Se vuelve a remarcar la naturaleza unitaria de la conducta en mérito a la cual los dominios se comprometen íntimamente. Sin embargo, el mérito de la categorización taxonómica reside en que especifica para el profesor y para el alumno los distintos énfasis y estrategias que conviene tener presentes según sea el objeto de un determinado aprendizaje. 
valorativo superior, que es el máximo cuidado de la salud de las personas.

Acerquémonos al momento en el cual el instructor y el alumno están a punto de iniciar un aprendizaje o de repetir un ejercicio de la clínica odontológica. El instructor observará que todas las condiciones se cumplan en cuanto a instrumental y ubicación del paciente y del operador frente al paciente, incluyendo el grado de iluminación que se precise. En este momento o en cualquier otro durante el procedimiento el instructor puede identificar alguna falta o imperfección, frente a esta observación su actitud puede ser de condescendencia: considera que la falta es menor y la deja pasar sin advertir al alumno, o puede estimular al alumno para que cuide y elimine la imperfección haciendo la corrección necesaria por pequeña que pueda ser. Este dilema que vive el instructor se irá repitiendo en cada uno de los momentos del proceso que merezcan su intervención, de modo que en adelante el alumno sea capaz de reconocer la falta o imperfección (cognición) y desarrolle la voluntad de buscar calidad en su actuación (acción positivamente intencionada)

Como quiera que durante el proceso de aprendizaje, particularmente en el dominio psicomotor, el alumno recibe el apoyo de varios instructores, estará expuesto a distintos niveles de exigencia de calidad, el resultado debe permitir obtener un balance aceptable que corresponderá a una serie de opciones de menor o mayor exigencia, una especie de rango de calidad aceptable dentro del cual cada alumno podrá ubicarse respondiendo a su carácter personal ${ }^{4}$.

Cabe remarcar que en el aprendizaje de la Odontología el nivel de exigencia alcanza tanto a procedimientos clínicos como aquellos de laboratorio y que, con el objetivo de alcanzar los mejores logros del proceso de aprendizaje, los instructores deben compartir el respeto a los estándares fijados por la institución docente.

Reconocemos además que desde las etapas más tempranas del aprendizaje la exigencia de calidad debe estar presente, considerando sin embargo que los niveles de logro de calidad son progresivos y que así mismo debe ser el nivel de exigencia hasta alcanzar los mayores niveles de perfección. El instructor debe evaluar el grado de aplicación de cada alumno para distinguir aquellas deficiencias que pueden atribuirse a lo temprano del aprendizaje, de las otras que responden a negligencia o falta de aplicación. Si en el primer caso el apoyo del profesor se dirigirá a facilitar el aprendizaje del procedimiento, en el segundo tendrá que ver con la modificación de la conducta, quizá con apoyo de consulta psicológica 5 .
Es indudable que en el desarrollo de la voluntad para el logro de calidad el alumno estará fuertemente influido por el ejemplo que le den sus instructores a través de su propia conducta. La institución universitaria debe disponer de un sistema permanente de supervisión de la actuación de los instructores a fin de sostener programas de educación continua y rotación en el ámbito de la enseñanza.

Para concluir diremos, que la educación de la voluntad es una obligación de la familia, del sistema educativo y de la sociedad en general respecto de la preparación para la vida de las futuras generaciones.

Ahora que se insiste tanto en la importancia de la educación para lograr el desarrollo de las naciones cabe remarcar la importancia de que ésta sea realmente integral. Sin la debida atención a la formación de la voluntad en el futuro ciudadano, será muy difícil que los operarios, artesanos, técnicos y profesionales cumplan a cabalidad en sus distintos roles sociales, incluido el quehacer político. Se trata en definitiva de la voluntad de cumplir con excelencia la tarea asumida por cada quien, como participante de un colectivo, $y$ perseverar en la búsqueda del Bien Común.

\footnotetext{
${ }^{4}$ Las variaciones que naturalmente corresponden a la personalidad de los instructores pueden reducirse en la práctica mediante ejercicios de calibración y preparación de manuales de procedimientos que detallen los componentes de la actividad en los niveles de: función, tarea, paso de tarea, movimiento y momento (Taxonomía de las Funciones Odontológicas de la Facultad de Estomatología de la Universidad Peruana Cayetano Heredia). Educación en Odontología -manual del profesor. Beltrán R. Fauno Editores SA, UPCH 1998 pp 142-156.

${ }^{5}$ Aunque en teoría el alumno que llega a la fase de aprendizaje clínico es porque el proceso educativo lo ha seleccionado al certificar sus conocimientos y disposición, no es raro encontrar alumnos en los niveles clínicos que carecen del grado de motivación suficiente como para empeñarse en un aprendizaje clínico de calidad. Creemos indispensable contar con el apoyo profesional de la psicología durante todos los años de los estudios odontológicos. Las primeras intervenciones en pacientes constituyen un salto difícil que puede requerir especial apoyo psicológico, de carácter preventivo para el conjunto de alumnos, así como recuperativo y rehabilitador para alumnos con problemas de adaptación al aprendizaje clínico.
} 